\title{
A Secondary Voltage Control Method for an AC/DC Coupled Transmission System Based on Model Predictive Control
}

\author{
Xu, Fengda; Guo, Qinglai; Sun, Hongbin; Wang, Bin; Wu, Qiuwei
}

Published in:

Proceedings of IEEE PES General Meeting 2015

Link to article, DOI:

10.1109/PESGM.2015.7286354

Publication date:

2015

Document Version

Publisher's PDF, also known as Version of record

Link back to DTU Orbit

Citation (APA):

Xu, F., Guo, Q., Sun, H., Wang, B., \& Wu, Q. (2015). A Secondary Voltage Control Method for an AC/DC Coupled Transmission System Based on Model Predictive Control. In Proceedings of IEEE PES General Meeting 2015 IEEE. https://doi.org/10.1109/PESGM.2015.7286354

\section{General rights}

Copyright and moral rights for the publications made accessible in the public portal are retained by the authors and/or other copyright owners and it is a condition of accessing publications that users recognise and abide by the legal requirements associated with these rights.

- Users may download and print one copy of any publication from the public portal for the purpose of private study or research.

- You may not further distribute the material or use it for any profit-making activity or commercial gain

- You may freely distribute the URL identifying the publication in the public portal 


\title{
A Secondary Voltage Control Method for an AC/DC Coupled Transmission System Based on Model Predictive Control
}

\author{
Fengda Xu, Qinglai Guo, Senior Member, IEEE, Hongbin Sun*, Senior Member, IEEE, Bin Wang, \\ Qiuwei Wu, Member, IEEE
}

\begin{abstract}
For an AC/DC coupled transmission system, the change of transmission power on the DC lines will significantly influence the AC systems' voltage. This paper describes a method to coordinated control the reactive power of power plants and shunt capacitors at DC converter stations nearby, in order to keep the voltage of the pilot bus tracking its set point considering the DC system's transmission schedule change. The approach is inspired by model predictive control (MPC) to compensate for predictable voltage change affected by DC side transmission power flow and the potential capacitor switching at DC converter stations. The control strategies are calculated from a multi-step dynamic optimization problem that is solved by mixed integer quadratic programming method. Time-domain simulations showed positive results of the proposed voltage controller.
\end{abstract}

Index Terms-HVDC system, Coordinated Voltage Control, Automatic Voltage Control, Model Predictive Control

\section{INTRODUCTION}

In China, in order to avoid sub-synchronous resonance and other problems, HVDC systems have been used to transmit large quantity of power energy between different large AC systems. Some DC converter stations at receiving side are located far from load center, where the network is very weak. The change of the DC lines' transmission schedule, which may cause large variation of power flow, will result in violent voltage fluctuation inside AC systems. At the receiving side of HVDC system between northeast China power grid and north China power grid, Gaoling converter station discussed in this paper is just an example.

Secondary voltage controller, which aims to keep the voltage of pilot buses close to their references and distribute reactive power appropriately ${ }^{[1-3]}$, has been widely applied in power system. The control interval is about 1-5 minutes that is restricted by communication conditions. However, traditional control method based on static optimization only concerns steady state information, so it doesn't always work well in an area containing DC converter stations since the shifting of DC operating mode could consequently lead to large active power flow changes. Furthermore, it is usually accompanied with

This work was supported in part by National Key Basic Research Program of China (973 Program) (2013CB228201), National Science Foundation of China (51277105) and the Foundation for Innovative Research Groups of the National Natural Science Foundation of China (51321005).

Hongbin Sun* is the corresponding author(e-mail: shb@tsinghua.edu.cn)

Fengda $\mathrm{Xu}$, Qinglai Guo, Hongbin Sun and Bin Wang are all with the Department of Electrical Engineering, State Key Laboratory of Power Systems, Tsinghua University, Beijing 100084, China.

Qiuwei Wu is with the Center for Electric Power and Energy, Department of Electrical Engineering, Technical University of Denmark, Kongens Lyngby 2800, Denmark, and also with the Sino-Danish Center for Education and Research, Aarhus 8000, Denmark automatic shunt capacitor switching inside the converter stations in only a few minutes, which may also cause reactive power change significantly and make the voltage much different from its last steady state.

The key to control voltage better in an area containing DC converter station at operating mode shifting period is to take the trend of transmission power flow and the consequent auto actions of the capacitors at the converter stations into consideration. Therefore, an MPC based method for secondary voltage control in an AC/DC coupled transmission system is proposed in this paper. MPC is an important method of process control theory, and has been applied to distribution networks voltage control [4-6], transmission voltage control [7], voltage stabilization under contingency [8-9], active power dispatch [10-11], energy storage management [12] and other fields and is showed to have good performance at coordinating different control units through the time.

The proposed method has some advantages comparing to the traditional one. Firstly, it concerns not only the present state but also the prediction states during a period of time in the future. That confirms an optimal control process of voltage. Secondly, predicted DC transmission power flow and behavior of shunt capacitors at converter stations are both considered, so it is possible to cooperate with power plants and the DC converter stations in the area. By the way, DC transmission power flow always changes according to a pre-defined schedule, so its predicted value could be exactly precise. Thirdly, voltage limits of all buses in the area are added to constraints, which assures the safety of the AC/DC system in a time-slot.

\section{MODEL PREDICTIVE CONTROL}

The proposed method concerns about the performance of system during $N$ control circles in the future, and each control circle contains $M$ predicted points as showed in Fig. 1. The value of DC power flow which changes according to predefined transmission schedule. Meanwhile, the behavior of shunt capacitors at the converter station also obeys fixed rules, which means a determined model can be built. 


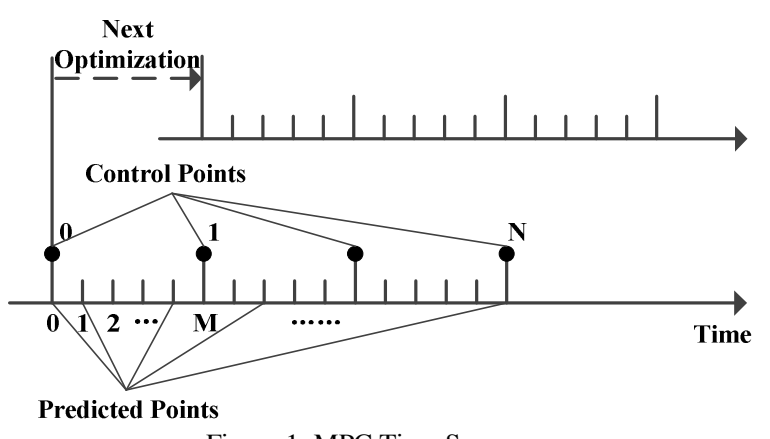

Figure 1. MPC Time Sequence

The first point of control sequence, the solution of optimization model, would be sent to each voltage control unit in the area according to MPC theory.

\section{A. Objective}

The voltage deviation of pilot buses is a key indicator of control system's performance. Thus the objective of optimization is showed as below.

$$
\min _{V_{\mathrm{G}}^{\mathrm{set}}} \sum_{i=0}^{N-1} \sum_{j=0}^{M-1} \rho^{t_{i, j}} F_{1}
$$

In the equation above, optimization variables $V_{G}^{\text {set }}$ is the set point of each generators' terminal voltage; $t_{i, j}=(M i+j) \Delta t$ represents for the $j$ th predicted point in the $i$ th control period, and $\Delta t$ is the interval between two predicted points; $\rho$ is a decay coefficient with a value less than 1; $F_{1}$ stands for deviations between predicted values and the references of pilot buses at the typical time $t_{i, j}$, and the specific expression is showed as below.

$$
F_{1}\left(t_{i, j}\right)=\sum_{k}\left[V_{\text {Pilot, } \mathrm{k}}^{\mathrm{pre}}\left(t_{i, j}\right)-V_{\text {Pilot, } \mathrm{k}}^{\mathrm{ref}}\right]^{2}
$$

$V_{\text {Pilot, } \mathrm{k}}^{\mathrm{pre}}$ and $V_{\text {Pilot,k }}^{\text {ref }}$ separately stand for the predicted voltage value and the reference of the $k$ th pilot bus.

\section{B. Constraints}

i) Voltage prediction can be obtained by solving power flow sensitivity equations.

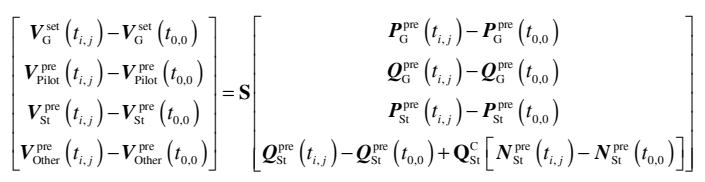

Here $\boldsymbol{V}_{\mathrm{G}}^{\text {set }}$ is the vector of generator terminal voltages' set values, $\boldsymbol{V}_{\text {Pilot }}^{\text {pre }}$ is the vector of predicted pilot buses' voltages, $\boldsymbol{V}_{\mathrm{St}}^{\text {pre }}$ is the vector of predicted converter stations' bus voltages, and $\boldsymbol{V}_{\text {Other }}^{\text {pre }}$ is the vector of other buses' voltages. $\mathbf{S}$ is the sensitivity matrix of bus voltage to injected power flow. $\boldsymbol{P}_{\mathrm{G}}^{\text {pre }}$ and $\boldsymbol{Q}_{\mathrm{G}}^{\mathrm{pre}}$ are separately the vector of predicted values of generators' active and reactive powers. $\boldsymbol{P}_{\mathrm{St}}^{\text {pre }}$ and $\boldsymbol{Q}_{\mathrm{St}}^{\mathrm{pre}}$ are that of DC transmission power flow, which can be precisely obtained according to their pre-defined transmission schedules. $\mathbf{Q}_{\mathrm{St}}^{\mathrm{C}}=\operatorname{diag}\left[\begin{array}{lll}Q_{\mathrm{St}, 1}^{\mathrm{C}} & Q_{\mathrm{St}, 2}^{\mathrm{C}} & \cdots\end{array}\right]$ represents the capacity of a single capacitor in converter stations, while $N_{\mathrm{St}}^{\text {pre }}$ stands for the number of capacitors connected to the network.

ii) In order to trigger the switching event of a capacitor, the voltage at the time just before the switching event should be obtained as a judgment. It can be deduced in the same way using sensitivity equations.

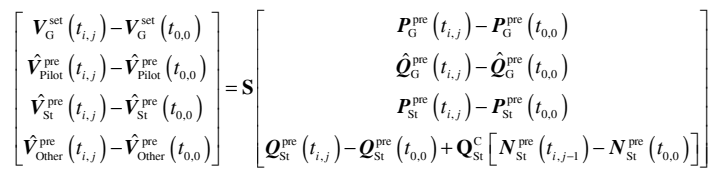

Here $\hat{\boldsymbol{V}}_{\text {Pilot }}^{\text {pre }}$ is the vector of predicted pilot buses' voltages, $\hat{V}_{\mathrm{St}}^{\text {pre }}$ is the vector of predicted converter stations' bus voltages, and $\hat{\boldsymbol{V}}_{\text {Other }}^{\text {pre }}$ is the vector of other buses' voltages at that time. $\hat{\boldsymbol{Q}}_{\mathrm{G}}^{\mathrm{pre}}$ is correspondingly the vector of predicted values of generators' reactive powers.

iii) Shunt capacitors' switching rules.

$$
N_{\mathrm{St}}^{\mathrm{pre}}\left(t_{i, j}\right)=\left\{\begin{array}{l}
N_{\mathrm{St}}^{\mathrm{pre}}\left(t_{i, j-1}\right)-1, \hat{V}_{\mathrm{St}}^{\mathrm{pre}}>V_{\mathrm{St}}^{\mathrm{max}} \\
N_{\mathrm{St}}^{\mathrm{pre}}\left(t_{i, j-1}\right)+1, \hat{V}_{\mathrm{St}}^{\mathrm{pre}}<V_{\mathrm{St}}^{\mathrm{min}} \\
N_{\mathrm{St}}^{\mathrm{pre}}\left(t_{i, j-1}\right), \text { else }
\end{array}\right.
$$

There is a set of rules for shunt capacitors at the converter station under voltage control mode. When the voltage of a converter station exceeds its upper or lower bound $V_{\mathrm{St}}^{\max } / V_{\mathrm{St}}^{\min }$, a capacitor will be put into or cut off from the grid. Besides, there are also rules under other kinds of station control mode such as reactive control mode, under which voltage bounds are taken place by reactive power bounds. Logical constraints in equation 5 can be transferred into mix integer form as showed below.

$$
\left\{\begin{array}{l}
N_{\mathrm{St}}^{\mathrm{pre}}\left(t_{i, j}\right)=N_{\mathrm{St}}^{\mathrm{pre}}\left(t_{i, j-1}\right)-C t_{\mathrm{St}}^{\mathrm{pre}}\left(t_{i, j}\right)+P t_{\mathrm{St}}^{\mathrm{pre}}\left(t_{i, j}\right) \\
\left(C t_{\mathrm{St}}^{\mathrm{pre}}-1\right) R<\hat{V}_{\mathrm{St}}^{\mathrm{pre}}-V_{\mathrm{St}}^{\mathrm{max}}<C t_{\mathrm{St}}^{\mathrm{pre}} R \\
\left(P t_{\mathrm{St}}^{\mathrm{pre}}-1\right) R<V_{\mathrm{St}}^{\mathrm{min}}-\hat{V}_{\mathrm{St}}^{\mathrm{pre}}<P t_{\mathrm{St}}^{\mathrm{pre}} R
\end{array}\right.
$$

$C t_{\mathrm{St}}^{\mathrm{pre}}$ and $P t_{\mathrm{St}}^{\mathrm{pre}}$ are both $0-1$ variables, while $R$ is a big positive number.

iv) Shunt capacitors in a converter station should not be frequently switched, since it may cause damage to both the capacitors and power system. Therefore, the constraints of switching times are set. 


$$
\left\{\begin{array}{l}
O_{\mathrm{St}}^{\mathrm{pre}}\left(t_{i, j}\right)=C t_{\mathrm{St}}^{\mathrm{pre}}\left(t_{i, j}\right)+P t_{\mathrm{St}}^{\mathrm{pre}}\left(t_{i, j}\right) \\
\sum_{i=0}^{N-1} \sum_{j=0}^{M-1} O_{\mathrm{St}}^{\mathrm{pre}}\left(t_{i, j}\right)_{1} \leq O_{\mathrm{St}}^{\max }
\end{array}\right.
$$

Apparently $O_{\mathrm{St}}^{\mathrm{pre}}$ is a $0-1$ variable representing the action of capacitors, while $O_{\mathrm{St}}^{\max }$ is the limit of switching times.

v) Operating limits of system including that of bus voltages, generator reactive powers and numbers of shunt capacitors in converter stations.

$$
\left[\begin{array}{l}
\boldsymbol{V}_{\mathrm{G}}^{\min } \\
\boldsymbol{V}_{\mathrm{Pilot}}^{\min } \\
\boldsymbol{V}_{\mathrm{St}}^{\min } \\
\boldsymbol{V}_{\mathrm{Other}}^{\min } \\
\boldsymbol{Q}_{\mathrm{G}}^{\text {min }} \\
\boldsymbol{N}_{\mathrm{St}}^{\min }
\end{array}\right] \leq\left[\begin{array}{l}
\boldsymbol{V}_{\mathrm{G}}^{\text {set }} \\
\boldsymbol{V}_{\text {Pilot }}^{\text {pre }} \\
\boldsymbol{V}_{\mathrm{St}}^{\text {pre }} \\
\boldsymbol{V}_{\text {Other }}^{\text {pre }} \\
\boldsymbol{Q}_{\mathrm{G}}^{\text {set }} \\
\boldsymbol{N}_{\mathrm{St}}^{\text {pre }}
\end{array}\right] \leq\left[\begin{array}{l}
\boldsymbol{V}_{\mathrm{G}}^{\max } \\
\boldsymbol{V}_{\text {Pilot }}^{\max } \\
\boldsymbol{V}_{\mathrm{St}}^{\max } \\
\boldsymbol{V}_{\text {Other }}^{\max } \\
\boldsymbol{Q}_{\mathrm{G}}^{\max } \\
\boldsymbol{N}_{\mathrm{St}}^{\max }
\end{array}\right]
$$

\section{Solving Algorithm}

Expressions 1-4 and 6-8 compose the whole optimizing model of the secondary voltage controller in the AC/DC coupled transmission system. It is a mix integer quadratic programming problem, which can be solved by dual simplex algorithm accompanied with branch-and-bound method.

\section{Simulation Results}

\section{A. Simulation System}

The simulation system is built based on the north China power grid, and use the history data of it in Oct. $25^{\text {th }} 2013$ as input data. The secondary voltage control with a 5 minute cycle is restricted to an area showed in Fig. 2. High voltage bus of TM Station is selected as the pilot bus. In the simulation, the reference value of pilot bus voltage is set to $520 \mathrm{kV}$, while the upper and lower voltage bounds that trigger switching events of capacitors at the converter station are separately $534 \mathrm{kV}$ and $518 \mathrm{kV}$. Parameters $N$ and $M$ are separately set to 2 and 5 , so that the MPC optimization time slot is 10 minute wide, and the interval of predictions $\Delta t$ is 1 minute.

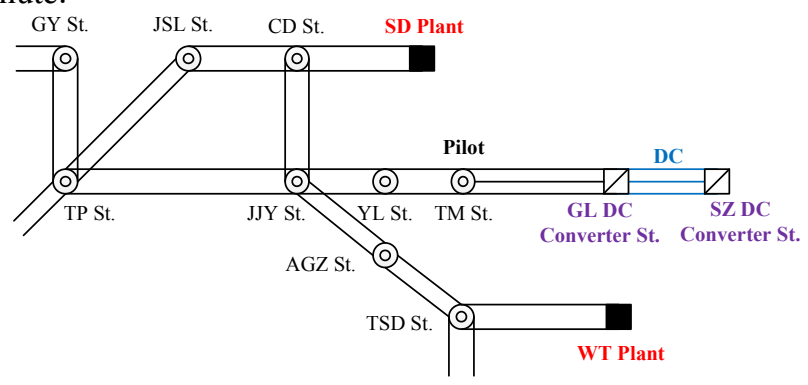

Figure 2. Secondary Voltage Control Area

Since the problem discussed doesn't refer to transient process, a power flow calculation program is used to simulate the changing states of the system. The solving of optimization model is carried out by CPLEX.

\section{B. DC Transmission Power Decreasing}

DC transmission power starts to decrease at 1:00 a.m. from about $3000 \mathrm{MW}$ to $2100 \mathrm{MW}$ at a speed of $-30 \mathrm{MW} / \mathrm{min}$, as showed in Fig. 3. This results in voltages of nearby buses' increasing and shunt capacitors' being cut down at the converter station.

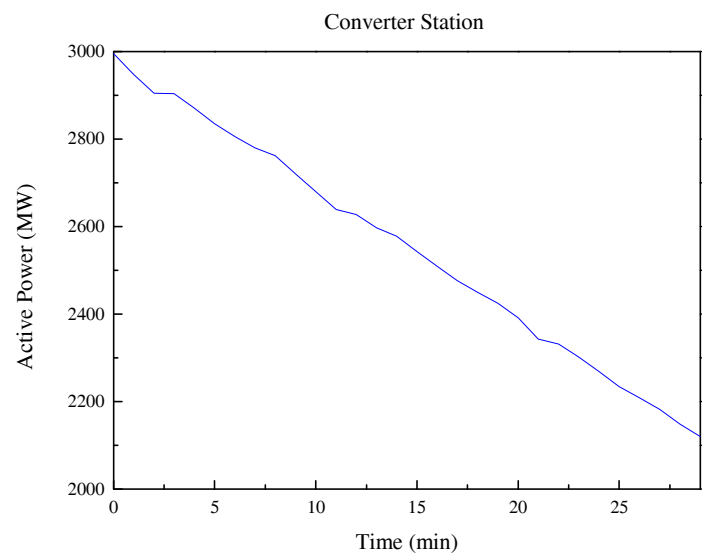

Figure 3. Active Power of DC Transmission Line

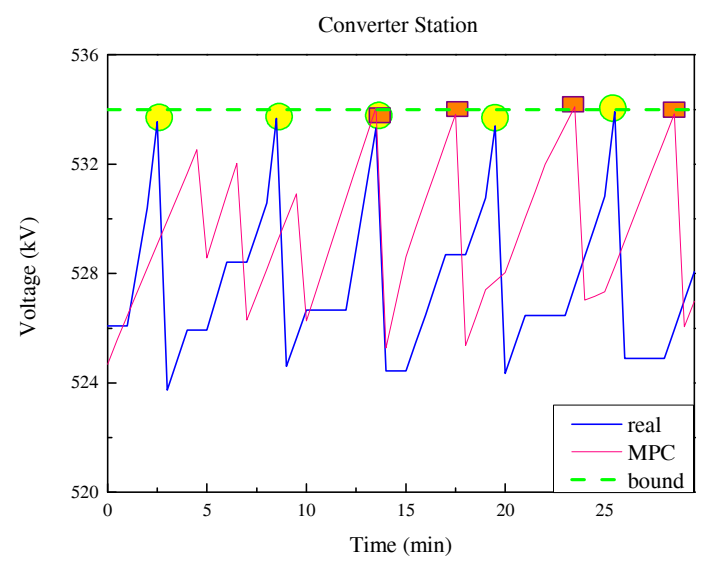

Figure 4. Converter Station Voltage 


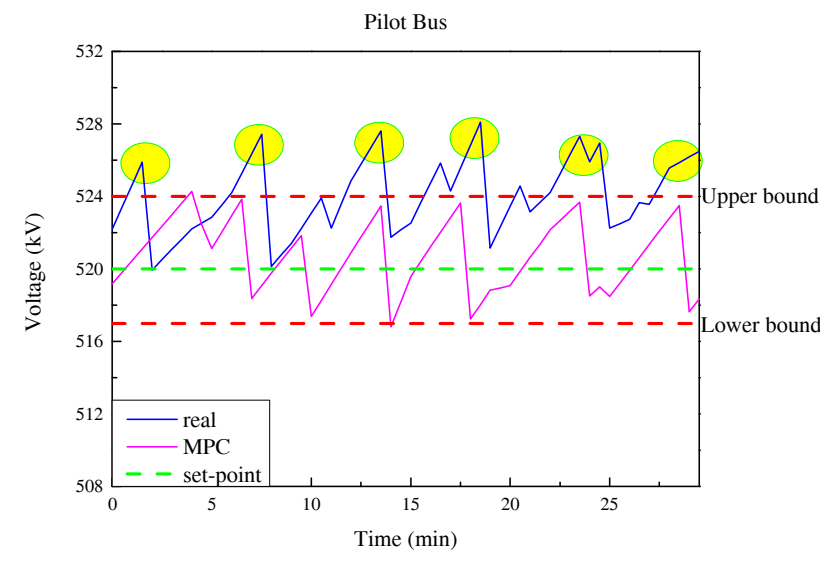

Figure 5. Pilot Bus Voltage

Fig. 4 and Fig. 5 separately show the voltage value of the converter station and the pilot bus. We can see in Fig. 4, yellow circles and orange squares stand for switching events under traditional and MPC controller. With the MPC based method, switching times of capacitors is lesser. Meanwhile, Fig. 5 illustrates that controller using proposed method keeps the voltage of pilot bus staying in the safe range, but the traditional controller doesn't always do (yellow circles). In fact with the MPC method, the system realizes that alongside with the decreasing of DC transmission power, voltage of the converter station has to reach its upper bound to trigger switching of capacitors. The voltage drop between the DC converter station and the pilot bus station is determined by the active and reactive power transferred between them which are strongly related with the DC power flow and the number of capacitors connected with the grid. The effect of DC power flow on voltage drop would more or less be offset regularly by capacitors. Thus, power plants will adjust their reactive power in advance to affect the switching time of capacitors, in order to enlarge the voltage drop and reduce the voltage value of the pilot bus at that time.

\section{DC Transmission Power Increasing}

DC transmission power starts to increase at 4:45 a.m. from about $2100 \mathrm{MW}$ to $3000 \mathrm{MW}$ at a speed of $30 \mathrm{MW} / \mathrm{min}$, as showed in Fig. 6. This results in voltages of nearby buses' decreasing and shunt capacitors' being put into the power grid at the converter station.

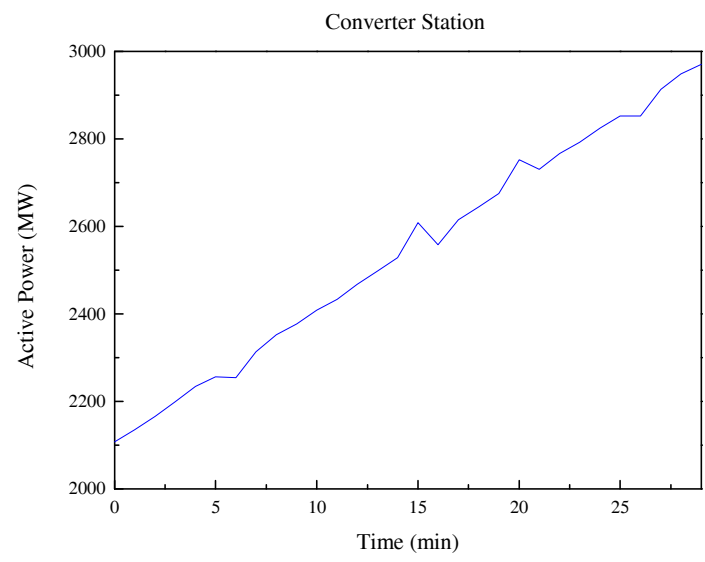

Figure 6. Active Power of DC Transmission Line

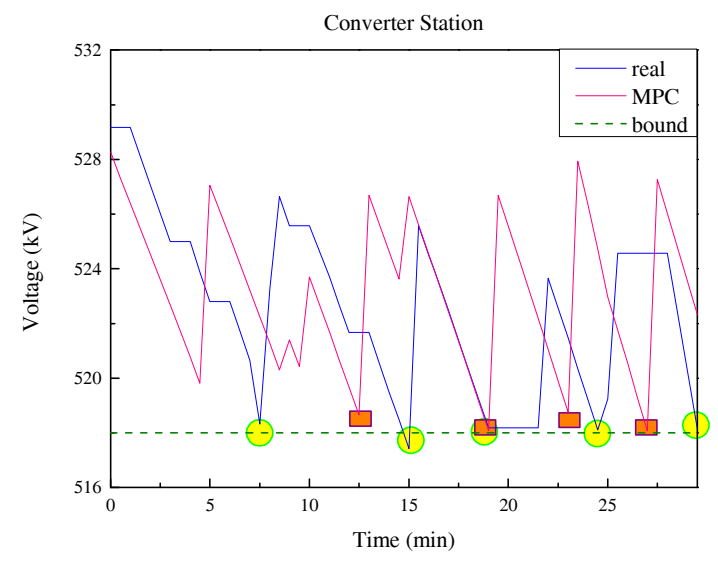

Figure 7. Converter Station Voltage

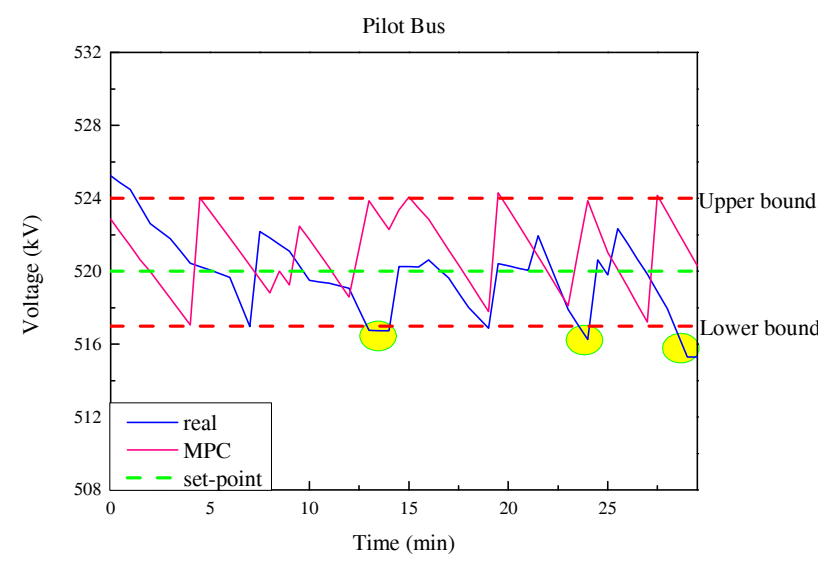

Figure 8. Pilot Bus Voltage

Fig. 7 and Fig. 8 separately show the voltage value of the converter station and the pilot bus. We can see in Fig. 7, yellow circles and orange squares stand for switching events 
under traditional and MPC controller. With the MPC based method, switching times of capacitors is lesser. Meanwhile, Fig. 8 illustrates that controller using proposed method keeps the voltage of pilot bus staying in the safe range, but the traditional controller doesn't always do (yellow circles). In fact with the MPC method, the system realizes that alongside with the increasing of DC transmission power, voltage of the converter station has to reach its lower bound to trigger switching of capacitors. Thus, power plants will adjust their reactive power in advance to affect the switching time of capacitors, in order to lessen the voltage drop and increase the voltage value of the pilot bus at that time.

\section{CONCLUSIONS}

An MPC based secondary voltage control method is proposed in this paper. Here, what we predict include two parts, first is the active power variation trend according to the pre-defined transmission schedule of DC lines, and second is the reactive power variation trend considering the consequent auto-actions of shunt capacitors at converter stations. Based on the proposed dynamic programming problem, the control performances not only for the current snapshot but also for a future time-slot are optimized. In an AC/DC coupled area, especially where the DC lines feed into a relatively weak AC areas, it is proved that the proposed voltage control method would assure better voltage profiles.

\section{REFERENCES}

[1] Paul J.P., Leost J.Y. and Tesseron J.M., "Survey of the Secondary Voltage Control in France: Present Realization and Investigations," Power Engineering Review, IEEE , vol.PER-7, no.5, pp.55-56, May 1987.

[2] Ilic-Spong M., Christensen J. and Eichorn K.L., "Secondary voltage control using pilot point information," Power Systems, IEEE Transactions on, vol.3, no.2, pp.660-668, May 1988.

[3] Janssens N., "Tertiary and secondary voltage control for the Belgian HV system," International Practices in Reactive Power Control, IEE Colloquium on , pp.8/1,8/4, 7 Apr 1993.

[4] Valverde, G. and T. Van Cutsem, "Model Predictive Control of Voltages in Active Distribution Networks," Smart Grid, IEEE Transactions on , vol.4, no.4, pp.2152-2161, Dec. 2013

[5] Moradzadeh, M., L. Bhojwani and R. Boel, "Coordinated voltage control via distributed model predictive control," Control and Decision Conference (CCDC), 2011 Chinese , pp.1612-1618, 23-25 May 2011.

[6] Moradzadeh, M., R. Boel and L. Vandevelde, "Voltage Coordination in Multi-Area Power Systems via Distributed Model Predictive Control," Power Systems, IEEE Transactions on , vol.28, no.1, pp.513-521, Feb. 2013.

[7] Glavic, M. and T. Van Cutsem, "Some Reflections on Model Predictive Control of Transmission Voltages," Power Symposium, 2006. NAPS 2006. 38th North American , pp.625-632, 17-19 Sept. 2006.

[8] Jin, L., R. Kumar and N. Elia, "Application of Model Predictive Control in Voltage Stabilization," American Control Conference, 2007. ACC '07 , pp.5916-5921, 9-13 July 2007.

[9] Licheng, J., R. Kumar and N. Elia, "Model Predictive Control-Based Real-Time Power System Protection Schemes," Power Systems, IEEE Transactions on , vol.25, no.2, pp.988-998, May 2010.

[10] Le Xie, X. Ilic and D. M, "Model predictive dispatch in electric energy systems with intermittent resources," Systems, Man and Cybernetics, 2008. SMC 2008. IEEE International Conference on , pp.42-47, 12-15 Oct. 2008

[11] Le Xie, X. Ilic and D. M, "Model predictive economic/environmental dispatch of power systems with intermittent resources," Power \& Energy Society General Meeting, 2009. PES '09. IEEE , pp.1-6, 26-30 July 2009.
[12] Khalid, M. and A.V. Savkin, "A model predictive control approach to the problem of wind power smoothing with controlled battery storage," Renewable Energy, 2010. vol.35, no.7, pp.1520-1526.

\section{BIOGRAPHIES}

Fengda Xu was born in Zhenjiang City, Jiangsu Province in China on Nov. 30, 1989. He obtained his B. Eng. in Electronic Engineering from Tsinghua University, Beijing, China, in 2012. He is currently studying towards his $\mathrm{PhD}$ in Automatic Voltage Control, specializing in Power System Automation at Tsinghua University (THU), Beijing, China. His current research interests include the automatic voltage control, integration of renewable generation on the power system, model predictive control and smart grids.

Qinglai Guo (SM'14) received the B.S. degree from the Department of Electrical Engineering, Tsinghua University, Beijing, China, in 2000 and the $\mathrm{Ph} . \mathrm{D}$. degree from Tsinghua University in 2005. He is now an Associate Professor at Tsinghua University. His special fields of interest include EMS advanced applications, especially automatic voltage control and V2G.

Hongbin Sun (SM'12) received the double B.S. degrees from Tsinghua University, Beijing, China, in 1992 and the Ph.D. degree from the Department of Electrical Engineering, Tsinghua University in 1997. He is now Changjiang Chair Professor of Education Ministry of China, Full Professor of electrical engineering in Tsinghua University and Assistant Director of State Key Laboratory of Power Systems in China. From 2007 to 2008, he was a Visiting Professor with School of Electrical Engineering and Computer Science, Washington State University, Pullman, WA, USA. In the last 15 years, he has developed a commercial system-wide automatic voltage control systems which has been applied to more than 20 large-scale power grids in China. He published more than 200 academic papers. He held more than 20 patents in China.

Prof. Sun is an IET Fellow, members of IEEE PES CAMS Cascading Failure Task Force and CIGRE C2.13 Task Force on Voltage/Var support in System Operations. He won the China National Technology Innovation Award for his contribution on successful development and applications of New Generation of EMS for Power Systems in 2008, the National Distinguished Teacher Award in China for his contribution on power engineering education in 2009, and the National Science Fund for Distinguished Young Scholars of China for his contribution on power system operation and control in 2010.

Bin Wang received his Bachelor degree from Dept. of Electrical Engineering at Tsinghua University in 2005 and his $\mathrm{PhD}$ degree from Tsinghua University in 2012. His research interests include automatic voltage control and multi-agent system.

Qiuwei Wu (M'08) received the B.Eng. and M.Eng. degrees from the Nanjing University of Science and Technology, Nanjing, China, in 2000 and 2003, respectively, both in power system and automation, and the Ph.D. degree in power system engineering from Nanyang Technological University, Singapore, in 2009.

He was a Senior R\&D Engineer with VESTAS Technology R\&D Singapore Pte, Ltd., from March 2008 to October 2009. He was a Postdoctoral Researcher with Centre for Electric Technology, Department of Electrical Engineering, Technical University of Denmark, Kongens Lyngby, Denmark, from November 2009 to October 2010 and was an Assistant Professor with the same centre from November 2010 to August 2013. He has been an Associate Professor with the same centre since September 2013. 